\title{
Ser Idoso, Vítima de Trauma, é Fator de Risco Para Mortalidade Na Terapia Intensiva: Mito ou Realidade?
}

\author{
${ }^{1}$ Genesis S Barbosa, ${ }^{2}$ Bruna RS Moura, ${ }^{3}$ Tatiane GGN do Rio, ${ }^{4}$ Sérgio D Martuchi, ${ }^{5}$ Kézia P Lima \\ ${ }^{6}$ Daniela V Silva, ${ }^{7}$ Regina MC Sousa, ${ }^{8}$ Lilia S Nogueira
}

\section{RESUMO}

Objetivos: Comparar idosos e não idosos, vítimas de trauma, segundo características sociodemográficas e clínicas e identificar se idade avançada ( $\geq 60$ anos) é fator de risco para mortalidade na Unidade de Terapia Intensiva (UTI).

Material e método: Coorte prospectiva, realizada entre 2012/2015, em quatro hospitais em São Paulo, Brasil. A amostra foi constituída por vítimas de trauma com idade $\geq 18$ anos e permanência superior a 24 horas na UTI. Foram comparados dois grupos: idosos ( $\geq 60$ anos) e não idosos ( $<60$ anos). Os testes Qui-Quadrado de Pearson, Exato de Fisher, t-Student e regressão logística múltipla foram aplicados, com nível de significância de $5 \%$.

Resultados: Das 380 vítimas (73 idosos e 307 não idosos), a maioria era homens $(87,9 \%)$, com idade média de $41,5( \pm 18,9)$ anos. A causa externa mais frequente foi queda $(34,7 \%)$ seguida de acidente motociclístico $(23,4 \%)$. A maioria dos idosos foi vítima de queda $(75,4 \%)$. O tempo médio de permanência na UTI foi de $14,9( \pm 15,6)$ dias e um total de $74(19,5 \%)$ pacientes evoluiu a óbito (53 não idosos e 21 idosos). Houve diferença significativa entre os grupos $(p<0,05)$ em relação ao gênero, causa externa, tipo de admissão na UTI, Injury Severity Score (ISS), New Injury Severity Score (NISS), Simplified Acute Physiology Score (SAPS II) e mortalidade. Os idosos apresentaram menores valores de ISS e NISS e maiores escores do SAPS II do que os não idosos. O fator de risco para mortalidade foi SAPS II (OR = 1,04; IC95\% 1,03-1,06; $p<0,001)$ e tempo de permanência na UTI foi identificado como fator de proteção para o desfecho (OR = 0,98; IC95\% 0,96-0,99; $p=0,021)$.

Conclusões: Apesar dos idosos apresentaram maior mortalidade do que os não idosos, a idade, variável de interesse desta pesquisa, não foi fator de risco para mortalidade em UTI.

Significado clínico: Os resultados deste estudo podem contribuir para estratégias de melhoria da qualidade do atendimento ao idoso traumatizado.

Palavras-chave: Ferimentos e lesões, Idosos, Mortalidade, Unidades de terapia intensiva.

\footnotetext{
${ }^{1}$ Research Scholar, ${ }^{2,4-6}$ Postgraduate Student, ${ }^{3}$ Nurse ${ }^{7,8}$ Professor

1-2,4-8 Department of Medical Surgical Nursing, University of São Paulo, São Paulo, Brazil

${ }^{3}$ Department of Medical Surgical Nursing, Hospital Samaritano São Paulo, Brazil

Corresponding Author: Genesis S Barbosa, Research Scholar, Department of Medical Surgical Nursing, University of São Paulo, São Paulo, Brazil, Phone: +551130617544, e-mail: genesis@usp.br
}

How to cite this article: Barbosa GS, Moura BRS, do Rio TGGN, Martuchi SD, Lima KP, Silva DV, Sousa RMC, Nogueira LS. Ser Idoso, Vítima de Trauma, é Fator de Risco Para Mortalidade Na Terapia Intensiva: Mito ou Realidade? Panam J Trauma Crit Care Emerg Surg 2017;6(2):81-89.

Source of support: Nil

Conflict of interest: None

\section{ABSTRACT}

Aim: To compare the elderly and non-elderly trauma victims according to sociodemographic and clinical characteristics and identify whether advanced age ( $\geq 60$ years) is risk factor for mortality in the Intensive Care Unit (ICU).

Materials and methods: Prospective cohort, performed between 2012-2015, in four hospitals in São Paulo, Brazil. The sample consisted of trauma victims with age $\geq 18$ years and ICU length of stay more than 24 hours. Two groups were compared: elderly ( $\geq 60$ years) and non-elderly ( $<60$ years) patients. The Pearson's Chi-square, Fisher's exact, and t-Student tests and multiple logistic regression were applied, with a significance level of $5 \%$.

Results: Of the 380 victims (73 elderly and 307 non-elderly), the majority was men (87.9\%), with average age of $41.5( \pm 18.9)$ years. The most common external cause was fall $(34.7 \%)$ followed by motorcycle accident $(23.4 \%)$. Most of the elderly was the victim of fall $(75.4 \%)$. The average of ICU length of stay was 14.9 ( \pm 15.6$)$ days and $74(19.5 \%)$ patients died (53 non-elderly and 21 elderly). There was no significant difference between the groups $(p<0.05)$ in relation to the gender, external cause, type of ICU admission, Injury Severity Score (ISS), New Injury Severity Score (NISS), Simplified Acute Physiology Score (SAPS II) and mortality. The elderly patients showed lower values of ISS and NISS and higher SAPS II scores than nonelderly. The risk factor for mortality was SAPS II (OR = 1.04; $95 \% \mathrm{Cl} 1.03-1.06 ; p<0.001)$ and the length of stay in ICU was identified as protection factor for the outcome (OR $=0.98 ; 95 \%$ Cl 0.96-0.99; $p=0.021$ ).

Conclusion: Although the elderly had higher mortality than non-elderly, the age, variable of interest of this research, it was not a risk factor for ICU mortality.

Clinical significance: The results of this study can contribute to strategies for improving the quality of care for traumatized elderly.

Keywords: Aged, Intensive care units, Mortality, Wounds and injuries.

\section{INTRODUÇÃO}

Atualmente, as causas externas representam 9\% do total de óbitos por ano no mundo. ${ }^{1}$ Neste grupo, há uma 
maior representatividade dos adultos jovens, isto porque a doença apresenta uma relação direta de dependência com os fatores de risco a que o indivíduo está exposto. ${ }^{2}$

No Brasil, as causas externas de morbidade e mortalidade, que inclui o doente traumatizado, é a terceira maior causa de óbitos, correspondendo a $12 \%$ do total de mortes registrado no país. ${ }^{1,3-4}$ Com o aumento da expectativa de vida e consequente inversão da pirâmide etária, os idosos têm incrementado as estatísticas referentes ao trauma, tanto ao que concerne a morbidade quanto a mortalidade.

Estudos ${ }^{3,5-8}$ têm mostrado diferenças entre as populações de idosos e não idosos traumatizados em relação à causa externa, tipo e gravidade da lesão traumática, presença de doenças associadas, tempo de internação hospitalar e complicações pós-traumáticas ocorridas na Unidade de Terapia Intensiva (UTI).

Na população menor que 60 anos há predomínio de agressões seguido de acidentes de transporte. Na população idosa ( $\geq 60$ anos), há predominância de outras causas externas de lesões acidentais, do qual se inclui as quedas da própria altura ${ }^{2,4-6}$ e os atropelamentos ${ }^{4}$. Cabe ressaltar que, nos idosos, quanto maior a faixa etária observa-se o aumento das quedas da própria altura e a redução dos casos de atropelamento. ${ }^{4}$

Com as complicações oriundas do mecanismo do trauma associadas à idade, o idoso tende a ficar mais tempo internado no hospital e ser admitido na UTI. ${ }^{6-7}$ Estudo italiano mostra que, com o aumento da idade, eleva-se a chance da vítima de trauma morrer na unidade crítica. ${ }^{3}$ Além disso, pesquisadores apontam que idosos apresentam, em um mesmo tipo de acidente, maior probabilidade às lesões mais graves do que não idosos, além de maior chance de morte ao considerar lesões de gravidade semelhante. ${ }^{8}$

Frente ao exposto, o objetivo deste estudo é comparar idosos e não idosos, vítimas de trauma, segundo características sociodemográficas e clínicas e identificar se idade avançada ( $\geq 60$ anos) é fator de risco para mortalidade na UTI.

\section{MÉTODOS}

Trata-se de uma pesquisa observacional, do tipo coorte prospectiva, realizada entre 2012 e 2015, em quatro hospitais, localizados na Grande São Paulo, Brasil, centros de referência para o atendimento de alta complexidade à vítima de trauma.

Foram incluídos no estudo os pacientes admitidos na UTI que responderam aos seguintes critérios de elegibilidade: ser vítima de trauma contuso, penetrante ou misto (contuso e penetrante); ter idade igual ou superior a 18 anos; permanecer por, no mínimo, 24 horas na unidade crítica; e concordar em participar da pesquisa por meio da assinatura do Termo de Consentimento Livre e Esclarecido. Nos casos em que a condição clínica do paciente impedia o entendimento do termo, o consentimento foi obtido com familiares e/ou representantes legais. Os pacientes readmitidos na UTI foram excluídos da casuística.

Dois grupos foram criados e comparados na pesquisa: idosos ( $\geq 60$ anos) e não idosos ( $<60$ anos). A gravidade do trauma foi mensurada pelos índices Injury Severity Score (ISS) ${ }^{9}$ e New Injury Severity Score (NISS) ${ }^{10}$ a partir de informações registradas no prontuário do paciente, no laudo de exames ou em fichas cirúrgicas. A gravidade fisiológica das vítimas foi identificada pelo Simplified Acute Physiology Score (SAPS II) ${ }^{11}$ e a carga de trabalho de enfermagem pelo Nursing Activities Score (NAS). ${ }^{12}$ Para o cálculo do índice SAPS II foram considerados os dados clínicos e laboratoriais referentes às primeiras 24 horas de internação do paciente na UTI. O NAS foi identificado a partir das intervenções de enfermagem requeridas pelos pacientes durante este período.

Os testes Qui-Quadrado de Pearson, Exato de Fisher e t-Student foram aplicados na comparação dos grupos (idosos versus não idosos). A regressão logística múltipla, método stepwise backward, foi aplicada para identificar os fatores de risco para mortalidade na casuística. O nível de significância adotado em todas as análises foi de 5\%.

A pesquisa recebeu parecer favorável do Comitê de Ética em Pesquisa (parecer $\left.n^{\circ} 464.035\right)$.

\section{RESULTADOS}

Participaram deste estudo 380 vítimas, sendo 73 idosos (19,5\%) e 307 não idosos (80,5\%), a maioria do gênero masculino $(87,9 \%)$, com idade média de $41,5( \pm 18,9)$ anos. A causa externa mais frequente foi queda $(34,7 \%)$ seguida de acidente motociclístico (23,4\%) e atropelamento (21,3\%). O tipo de admissão na UTI foi cirúrgico na maioria da amostra (71,3\%); porém, somente em 7,1\% dos casos as cirurgias foram eletivas. Em relação à procedência, 67,1\% dos participantes veio diretamente do Centro Cirúrgico, $25,8 \%$ do Pronto Socorro e os demais foram transferidos de unidades de internação, UTI e outros hospitais. O tempo médio de permanência na UTI foi de $14,9( \pm 15,6)$ dias, a média do risco de morte pelo SAPS II no primeiro dia de internação foi de 23,9 $( \pm 9,8)$ e do NAS de 76,6 $( \pm 20)$. O ISS e o NISS médios foram de $17,8( \pm 8,3)$ e $23,9( \pm 9,8)$, respectivamente, e a mortalidade na UTI de $19,2 \%$.

Na Tabela 1, observa-se que houve diferença significativa entre os grupos em relação ao gênero $(p<0,001)$, causa externa $(p<0,001)$, tipo de trauma $(p=0,002)$ e tipo de admissão na UTI $(p<0,001)$. As mulheres, as quedas e as cirurgias programadas foram mais frequentes entre os idosos do que entre o grupo dos mais jovens. As quedas foram a causa externa predominante entre os idosos 
Tabela 1: Comparações entre idosos e não idosos em relação a gênero, causa externa, tipo de admissão na UTI e procedência. São Paulo, 2012-2015

\begin{tabular}{|c|c|c|c|c|c|}
\hline \multirow[b]{3}{*}{ Características } & \multicolumn{4}{|c|}{ Idoso } & \multirow[b]{3}{*}{ valor de $p$} \\
\hline & \multicolumn{2}{|c|}{ Sim } & \multicolumn{2}{|c|}{ Não } & \\
\hline & $n$ & $\%$ & $n$ & $\%$ & \\
\hline \multicolumn{6}{|l|}{ Gênero } \\
\hline Feminino & 34 & 46,6 & 37 & 12,1 & $<0,001^{*}$ \\
\hline Masculino & 39 & 53,4 & 270 & 87,9 & \\
\hline \multicolumn{6}{|l|}{ Causa Externa } \\
\hline Queda & 55 & 75,4 & 77 & 25,1 & $<0,001^{* *}$ \\
\hline Acidente Motociclístico & 2 & 2,7 & 87 & 28,3 & \\
\hline Acidente Automobilístico & 1 & 1,4 & 26 & 8,5 & \\
\hline Atropelamento & 12 & 16,4 & 69 & 22,5 & \\
\hline Outras & 3 & 4,1 & 48 & 15,6 & \\
\hline \multicolumn{6}{|l|}{ Tipo de trauma } \\
\hline Contuso & 73 & 100,0 & 273 & 88,9 & $0,002^{\star *}$ \\
\hline Penetrante & - & - & 34 & 11,1 & \\
\hline \multicolumn{6}{|l|}{ Tipo de admissão na UTI } \\
\hline Cirúrgica não programada & 39 & 53,4 & 205 & 66,8 & $<0,001^{*}$ \\
\hline Cirúrgica programada & 18 & 24,7 & 9 & 2,9 & \\
\hline Clínica & 16 & 21,9 & 93 & 30,3 & \\
\hline \multicolumn{6}{|l|}{ Procedência } \\
\hline Centro Cirúrgico & 55 & 75,3 & 200 & 65,1 & $0,249^{* *}$ \\
\hline Pronto Socorro & 14 & 19,2 & 84 & 27,4 & \\
\hline Outras & 4 & 5,5 & 23 & 7,5 & \\
\hline
\end{tabular}

*Teste Qui Quadrado; **Teste Exato de Fisher

$(75,4 \%)$ e todos os pacientes com idade $\geq 60$ anos foram vítimas de trauma contuso.

$\mathrm{Na}$ Tabela 2, nota-se que a gravidade do trauma segundo ISS e NISS diferiu entre os grupos $(p<0,001)$, assim como o risco de morte das vítimas na admissão na UTI pelo SAPS II $(\mathrm{p}<0,001)$. No entanto, os idosos apresentaram menores valores de ISS e NISS, e maiores escores do SAPS II, isto é, tiveram indicação de trauma mais leve e condições fisiológicas mais graves na admissão na UTI do que o grupo de não idosos.

Verificou-se diferença significativa $(\mathrm{p}=0,026)$ na comparação da mortalidade dos dois grupos (Tabela 3). A mortalidade entre os idosos foi aproximadamente $10 \%$ maior do que o outro grupo ( $28,8 \%$ perante a $17,3 \%)$.

A Tabela 4 mostra que o tempo de permanência na UTI e o risco de morte, segundo SAPS II alcançaram nível de significância para permanecer no modelo final. O acréscimo de um ponto no risco de morte pelo SAPS II na admissão na UTI aumentou em $4 \%$ a chance da vítima de trauma morrer durante a internação nessa unidade. O tempo de permanência foi identificado como fator de proteção para óbito e o aumento de um dia de internação na UTI, diminuiu a chance de morrer na unidade em $2 \%$.

Ainda que a mortalidade tenha sido mais expressiva entre o grupo de pacientes idosos, a idade, como variável dicotômica (<60 e 60+), não permaneceu no modelo final de regressão. Na análise conjunta das variáveis analisadas
Tabela 2: Estatísticas descritivas e comparações entre idosos e não idosos em relação ao tempo de permanência na UTI, ISS, NISS, SAPS II e NAS. São Paulo, 2012-2015

\begin{tabular}{|c|c|c|c|}
\hline \multirow[b]{2}{*}{ Características } & \multicolumn{2}{|c|}{ Idoso } & \multirow[b]{2}{*}{ valor de $p^{*}$} \\
\hline & Sim & Não & \\
\hline \multicolumn{4}{|c|}{$\begin{array}{l}\text { Tempo de permanência } \\
\text { na UTI }\end{array}$} \\
\hline Média & 13,8 & 15,1 & 0,504 \\
\hline Desvio padrão & 13,5 & 16,1 & \\
\hline Mediana & 11,0 & 11,0 & \\
\hline Mín-Máx & $0-56$ & $0-97$ & \\
\hline \multicolumn{4}{|l|}{ ISS } \\
\hline Média & 13,1 & 18,9 & $<0,001$ \\
\hline Desvio padrão & 5,4 & 8,4 & \\
\hline Mediana & 10,0 & 17,0 & \\
\hline Mín-Máx & $6-34$ & $4-50$ & \\
\hline \multicolumn{4}{|l|}{ NISS } \\
\hline Média & 18,0 & 25,4 & $<0,001$ \\
\hline Desvio padrão & 7,5 & 9,7 & \\
\hline Mediana & 17,0 & 27,0 & \\
\hline Mín-Máx & $9-43$ & $9-66$ & \\
\hline \multicolumn{4}{|c|}{ SAPS II (risco de morte) } \\
\hline Média & 36,2 & 23,2 & $<0,001$ \\
\hline Desvio padrão & 24,7 & 22,1 & \\
\hline Mediana & 32,6 & 15,3 & \\
\hline Mín-Máx & $1,0-92,5$ & $0,1-98,7$ & \\
\hline \multicolumn{4}{|l|}{ NAS } \\
\hline Média & 74,4 & 77,2 & 0,277 \\
\hline Desvio padrão & 20,4 & 19,9 & \\
\hline Mediana & 73,7 & 73,1 & \\
\hline Mín-Máx & $36-136$ & $39-143$ & \\
\hline
\end{tabular}


Tabela 3: Comparação da mortalidade em UTI de idosos e não idosos. São Paulo, 2012-2015

\begin{tabular}{llllll}
\hline & \multicolumn{4}{c}{ Idoso } & \\
\cline { 2 - 5 } & Sim & \multicolumn{3}{c}{ Não } & \\
\cline { 2 - 5 } Óbito na UTI & $n$ & $\%$ & $n$ & $\%$ & valor de $p^{*}$ \\
\hline Sim & 21 & 28,8 & 53 & 17,3 & 0,026 \\
Não & 52 & 71,2 & 254 & 82,7 & \\
\hline
\end{tabular}

*Teste Qui Quadrado

Tabela 5: Variáveis que não permaneceram no modelo de regressão logística para óbito de vítimas de trauma na UTI, segundo nível de significância. São Paulo, 2012-2015

\begin{tabular}{ll}
\hline Variáveis & Valor de $p$ \\
\hline Idade $(<60$ e 60+) & 0,657 \\
Gênero & 0,321 \\
Causa Externa & 0,377 \\
Tipo de admissão & 0,850 \\
Procedência & 0,687 \\
ISS & 0,146 \\
NISS & 0,199 \\
NAS & 0,953 \\
\hline
\end{tabular}

neste estudo, ser idoso não foi fator de risco para morrer na UTI após um evento traumático (Tabela 5).

\section{DISCUSSÃO}

Na casuística analisada, as mulheres e as quedas foram mais frequentes entre os idosos do que os não idosos, corroborando com outros autores que analisaram vítimas de trauma. ${ }^{8,13}$ A queda, ${ }^{6-8,13}$ especialmente do mesmo nível, ${ }^{7,4}$ é apontada como a causa externa mais importante entre idosos e tal fato pode estar associado às limitações de movimentos, diminuição de reflexos e problemas visuais e auditivos inerentes ao processo de envelhecimento. ${ }^{8,13}$ Além disso, o gênero feminino também tem sido relatado como um fator de risco para a ocorrência deste tipo de trauma, ${ }^{13-14}$ especialmente quando associado ao uso de medicamentos de uso contínuo $^{13}$ ou polifarmácia. ${ }^{14}$

Destaca-se neste estudo que os idosos foram submetidos, com maior frequência, a cirurgias programadas do que não idosos. Pesquisa que analisou 3112 vítimas de trauma identificou que as fraturas de extremidades prevaleceram entre idosos. ${ }^{7}$ Essas lesões, embora muitas vezes consideradas graves, permitem que manobras de estabilização clínica do paciente sejam realizadas antes do ato cirúrgico. Além disso, estudo brasileiro ${ }^{8}$ mostrou que procedimentos cirúrgicos de urgência, como drenagem de tórax e laparotomia exploratória, foram realizados em menor frequência nos idosos traumatizados quando comparados aos não idosos. Esses achados podem estar relacionados aos resultados observados na pesquisa em relação às cirurgias programadas.
Tabela 4: Modelo de regressão logística para óbito de vítimas de trauma na UTI. São Paulo, 2012-2015

\begin{tabular}{lllll}
\hline & & \multicolumn{2}{l}{ IC para OR (95\%) } \\
\cline { 3 - 5 } Variáveis & valor de $p$ & OR & $\begin{array}{l}\text { Limite } \\
\text { inferior }\end{array}$ & $\begin{array}{l}\text { Limite } \\
\text { superior }\end{array}$ \\
\hline Tempo de permanência & 0,021 & 0,98 & 0,96 & 0,99 \\
na UTI (dias) & & & & \\
SAPS II (risco de morte) & $<0,001$ & 1,04 & 1,03 & 1,06 \\
\hline
\end{tabular}

Os resultados deste estudo indicaram que os idosos apresentaram trauma mais leve e condições fisiológicas mais graves (maior risco de morte) na admissão na UTI do que o grupo de não idosos. Pesquisa que analisou 2075 traumatizados admitidos na sala de emergência mostrou que não houve diferença significativa entre idosos e não idosos em relação à gravidade do trauma mensurada pelo ISS. ${ }^{8}$ Vale salientar que este estudo não abordou especificamente pacientes que necessitaram de cuidados intensivos. Neste sentido, pode-se supor que os idosos necessitam de internação na UTI devido à instabilidade clínica decorrente do trauma associada às comorbidades e não necessariamente pela gravidade das lesões traumáticas.

$\mathrm{Na}$ análise da mortalidade hospitalar de vítimas de trauma, pesquisas mostram que idosos e não idosos não diferem em relação a este desfecho. ${ }^{7-8}$ Entretanto, quando se analisa a mortalidade na UTI, os resultados mostram que esta é significativamente diferente entre os grupos, com maior frequência de mortes entre os idosos, ${ }^{3}$ corroborando com a presente investigação.

Apesar da expressiva frequência de mortes entre os idosos, a idade não foi preditora de mortalidade na UTI entre as vítimas analisadas. O índice SAPS II destacouse como fator de risco para o desfecho estudado, assim como em outra investigação. ${ }^{15} \mathrm{O}$ escore SAPS II ${ }^{11}$ é obtido a partir da análise de 12 variáveis fisiológicas, além de idade, tipo de admissão e presença de doença crônica, sumarizando, em um único indicador, os aspectos fisiológicos que mais impactam no risco de morte do paciente e que estão em maior frequência na população de idosos. Ressalta-se que a idade é contemplada no índice SAPS II (quanto maior a idade, maior o escore) e isto pode ter contribuído para que a mesma não tenha se destacado, isoladamente, como fator preditor de mortalidade.

Estudos $^{15-17}$ tem mostrado que as mortes na UTI acontecem precocemente, ou seja, quando maior o tempo de permanência na unidade crítica, menor a chance do paciente morrer. Estes dados reforçam o achado desta pesquisa em que o tempo de permanência foi um fator de proteção para a mortalidade das vítimas de trauma.

A indicação de internação na UTI dos idosos desta pesquisa foi, provavelmente, relacionada às alterações fisiológicas apresentadas que exigem monitoramento 
intensivo e não à gravidade das lesões traumáticas. Nesse sentido, o monitoramento precoce dos idosos traumatizados deve ser considerado na melhoria da qualidade da assistência prestada a esta população.

Resultado de pesquisa realizada com dados do National Trauma Data Bank sugere que a idade superior a 60 anos deve ser um critério para o encaminhamento das vítimas de trauma para hospitais preparados para atendimento de alta complexidade, uma vez que esses pacientes apresentam maior morbidade e mortalidade do que jovens, independente do nível de gravidade. ${ }^{18}$ Esses achados reforçam os resultados deste estudo que destacam as alterações fisiológicas do idoso traumatizado como fator de risco para mortalidade na UTI, indicando a necessidade de atendimento em hospitais especializados.

\section{CONCLUSÃO}

Os idosos e não idosos diferiram em relação a algumas características sociodemográficas e clínicas, com destaque à gravidade das lesões traumáticas e das condições fisiológicas. Idosos tiveram indicação de trauma mais leve e condições fisiológicas mais graves na admissão na UTI do que o grupo de não idosos. Ainda que a mortalidade tenha sido mais expressiva entre o grupo de pacientes idosos, a análise da idade em conjunto com outras variáveis do estudo mostrou que ser idoso não foi fator de risco para morrer na UTI após um evento traumático.

\section{REFERÊNCIAS}

1. World Health Organization. Violence and Injuries: the facts. Geneva, Switzerland, 2010.

2. Imamura JH, Troster EJ. Epidemiologia dos traumas e países desenvolvidos e em desenvolvimento. Dissertação (Mestrado). Faculdade de Medicina da Universidade de São Paulo, 2012. Pg. 6; 11-17.

3. Mondello S, Cantrell A, Domenico I, Fodale V, Mondello P, Ang D. Complications of Trauma Patients Admitted to the ICU in Level I Academic Trauma Centers in the United States. Biomed Res Int 2014;.

4. Ministério da Saúde do Brasil. Departamento de Informática do Sistema Único de Saúde do Brasil (DATASUS). Informações de Saúde: Estatísticas Vitais. Brasília, 2016. Acesso em 30/09/2016 < http://www2.datasus.gov.br/DATASUS/ index.php?area $=0205 \&$ id $=6937>$

5. Aguiar Júnior W, Saleh CMR, Whitaker IY. Risk Factors for Complications of Traumatic Injuries. J Trauma Nurs 2016 Sep-Oct;23(5):275-283. 6. Degani CC. Trauma em Idosos: Características e Evolução. Dissertação (Mestrado). Universidade de São Paulo, Ribeirão Preto; 2011. pp.71-98.

7. Broska Júnior CA, Del Folchini AB, Ruediger RR. Comparative study of trauma in the elderly and non-elderly patients in a University Hospital in Curitiba. Rev Col Bras Cir 2013;40(4):281-286.

8. Parreira JG, Soldá SS, Perlingeiro JAG, Padonese CC, Karakhanian WZ, Assef JC. Análise comparativa das características do trauma entre pacientes idosos e não idosos. Rev Assoc Med Bras 2010;56(5):541-546

9. Baker SP, O`Neill B, Haddon W, Long WB. The injury severity score: a method for describing patients with multiple injuries and evaluating emergency care. J Trauma 1974 Mar;14(3): 187-196.

10. Osler T, Baker SP, Long W. A modification of the injury severity score that both improves accuracy and simplifies scoring. J Trauma 1997 Dec;43(6):922-925.

11. Le Gall JR, Lemeshow S, Saulnier F. A new Simplified Acute Physiology score (SAPS II) based on a European/ North American multicenter study. JAMA 1993 Dec;270(24): 2957-2963.

12. Miranda DR, Nap R, Rijk A, Schaufeli W, Iapichino G. Nursing activities score. Crit Care Med 2003 Feb;31(2):374-382.

13. Rodrigues J, Ciosak SI. Elderly victims of trauma: analysis of the risk factors. Rev Esc Enferm USP 2012 Dec; 46(6):1 400-1405.

14. Buatois $S$, Perret-Guillaume C, Gueguen R, Miget $P$, Vançon G, Perrin P, Benetos A. A simple clinical scale to stratify risk of recurrent falls in community-dwelling adults aged 65 years and older. Phys Ther 2010 Apr;90(4):550-560.

15. Sardinha DS, de Sousa RM, Nogueira LS, Damiani LP. Risk factors for the mortality of trauma victims in the intensive care unit. Intensive Crit Care Nurs 2015 Apr;31(2):76-82.

16. Chalya PL, Gilyoma JM, Dass RM, Mchembe MD, Matasha M, Mabula JB, Mbelenge N, Mahalu W. Trauma admissions to the Intensive Care Unit at a reference hospital in Northwestern Tanzania. Scand J Trauma Resusc Emerg Med 2011 Oct;19:61.

17. Auñón Martín I, Caba Doussoux P, Mora Sambricio A, Guimera García V, Yuste García P, Resines Erasun C. Costanalysis of treating patients with multiple injuries in a reference hospital in Spain. Cir Esp 2012 Nov;90(9):564-568.

18. Shifflette VK, Lorenzo M, Mangram AJ, Truitt MS, Amos JD, Dunn EL. Should age be a factor to change from a level II to a level I trauma activation? J Trauma 2010 July;69(1):88-92. 


\section{INVITADO COMENTARIO}

\section{Ser idoso, vítima de trauma, é fator de risco para mortalidade na terapia intensiva: mito ou realidade?}

Uno de los mayores desafíos que el cirujano de trauma puede encontrar es el manejo agudo del paciente geriátrico. La población que presenta una edad mayor de los 60 años está expuesta a situaciones médico-quirúrgicas específicas que requieren un abordaje disímil en su manejo y tratamiento al compararse con una población adulta más joven. Esta población geriátrica se encuentra en un aumento considerable a nivel mundial. En los Estados Unidos, por ejemplo, ya constituye más del $13 \%$ de la población con un pronóstico que para el año 2020 constituya el $16 \%$ y para el año 2050 constituya el 20\% o una quinta parte de la población. ${ }^{1,2}$ Actualmente, trauma y sepsis son unas de las principales causas de muertes dentro de la población geriátrica en los Estados Unidos. En un estudio realizado en un centro de trauma de nivel uno, se encontró que, dentro de un periodo de doce años, el porcentaje de pacientes de trauma geriátricos admitidos a la institución hospitalaria presentó un aumento del $13 \%$ a un $27 \%$ del total de admisiones de los pacientes de trauma. Esto se reflejó en un aumento actual en el número total de admisiones de un $67 \% .{ }^{3}$ El manejo agudo del paciente geriátrico de trauma presenta consideraciones específicas para el profesional de la salud. Las lesiones en esta población son de origen multifactorial y hay que tomar en consideración las dificultades fisiológicas, peligros del medio ambiente y alteraciones en comportamiento relacionados al trauma del paciente. El área en donde se interceden estas tres condiciones presenta el grupo con el mayor índice de riesgo a sufrir una lesión. La causa más común de las visitas de los pacientes geriátricos de trauma a la sala de emergencia son las caídas. Entre un 65 al 70\% de las admisiones a un centro de trauma de parte de esta población son debido a caídas. ${ }^{4}$ En un estudio, los pacientes geriátricos mayores de 65 años de edad presentaron una probabilidad del 33\% de sufrir una caída y una probabilidad del $50 \%$ después de los 80 años. ${ }^{5}$ Además, una quinta parte de las fatalidades debido a accidentes vehículo-peatonal ocurren en víctimas mayores de los 65 años de edad. ${ }^{6}$

¿Cuál es la diferencia entre el manejo de pacientes geriátricos con urgencias quirúrgicas por trauma y pacientes de edad más joven? Condiciones médicas pre-existentes, una reserva fisiológica comprometida o disminuida, una capacidad funcional diaria disminuida, posible deterioro en la capacidad mental, intervenciones quirúrgicas previas, múltiples medicamentos, abuso doméstico, desconocimiento de las necesidades específicas de esta población por los profesionales de la salud y diferentes patrones de lesiones constituyen desafíos en el manejo de esta población. No solamente presentan cambios característicos anatómicos y fisiológicos, además, la mayoría de estos pacientes toman múltiples y variados medicamentos concomitantemente. No solamente presentan cambios característicos anatómicos y fisiológicos, además, la mayoría de estos pacientes toman múltiples y variados medicamentos concomitantemente. Los medicamentos más comunes son para controlar la presión arterial alta (55\%), antiarrítmicos $(26 \%)$, para el control de la glucosa sanguínea $(22 \%)$, antiplaquetarios $(22 \%)$ y anticoagulantes $(10 \%){ }^{7}$

La evaluación del paciente geriátrico se debe de hacer de la manera más oportuna al llegar a la sala de emergencia ya que para esta población médica la intervención inmediata es imperativa debido a sus condiciones médicas pre-existentes, pobre reserva fisiológica y estado nutricional deficiente. Uno de los factores más importantes que se deben evitar durante la evaluación de esta población es el subtriaje de los pacientes con un índice de severidad de lesión menor o moderada. Un alto grado de sospecha es necesario en el manejo del paciente geriátrico de trauma. El cuidado médico ofrecido al paciente geriátrico debe ser diferente al cuidado que se provee a la población más joven con el mismo tipo de lesiones traumáticas. ${ }^{8}$ Se ha apreciado que la atención y monitoreo detallado, minucioso y precoz de estos pacientes disminuyen el riesgo de mortalidad en un $4.2 \%{ }^{4}$

Uno de los factores más importantes en el manejo del paciente de trauma geriátrico es mantener un alto nivel de monitoreo médico a través de la estadía hospitalaria. Un cuidado agresivo y constante aumentara las probabilidades en estos pacientes de regresar al mismo nivel funcional físico y mental previo al evento traumático. ${ }^{9}$ Este es la meta primordial del equipo de trauma.

Una de las consideraciones primordiales en el manejo de los pacientes de trauma geriátrico es apreciar que estos pacientes presentan un mayor riesgo de mortalidad y morbilidad en comparación a la población adulta más joven con lesiones análogas. Un estudio encontró que pacientes con índices similares de severidad entre la población joven y la geriátrica, la población geriátrica con índices de severidad de lesiones leves y moderadas presentaba un aumento de la mortalidad de cinco veces y de cuatro veces con índices severos. ${ }^{8}$ Además, lo mismo ocurre con la morbilidad dentro de esta población. La población geriátrica presentó un aumento tres veces mayor entre los índices 
de severidad leves y moderados y de dos veces mayor entre los índices severos en comparación a la población adulta más joven. ${ }^{8}$ Por esta razón, los pacientes de trauma geriátricos deben ser evaluados, diagnosticados y manejados inmediatamente o tan pronto sea posible a su llegada a la sala de emergencia aun cuando presenten lesiones de leve o moderada intensidad. El subtriaje debe ser evitado a toda costa dentro de esta creciente población de pacientes.

Exhortamos a los autores a que continúen sus estudios y evaluaciones en esta población en específico con el propósito de mejorar su porcentaje de sobrevida y función independiente luego del trauma.

\section{REFERENCES}

1. Ortman, Jennifer M., et al. An Aging Nation: The Older Population in the United States. May 2014. p 25-1140. www.census.gov/ prod/2014pubs/p.25-1140.pdf

2. United Nations. Department of Economics an Social Affairs, populationdivision(2013).ST/ESA/SER.A/348.www.un.org/en/ development/desa/population/publications/pdf/ageing/world population ageing2013.pdf

3. Mangram AJ, Mitchell CD, Shifflette VK, Lorenzo M, Truitt MS, Goel A, Lyons MA, Nichols DJ, Dunn EL. Geriatric trauma service: a one-year experience. Published in the Journal of Trauma and Acute Care Surgery, Jan 2012; Vol 72 (1): p. 119-122.

4. Lorenzo M, Barba C, et al. Will Patient Outcomes Improve If We Admit and Manage Geriatric Patients in a Specialized Unit in the Trauma Center? Western Trauma Association Abstract Presentation. Jackson Hole, Wyoming 2005.

5. Schwab, CW, Kaunder DR, et al. Trauma in the Geriatric Patient. Arch Surg 1992;127:701.

6. Hannan EL, et al. Elderly Trauma in Patients in NY State: 1994-1998. J Trauma. 2004 Jun; 56(6):1297-1304.

7. McMahon DJ, Schawb CW, Kaunder DR. Comorbidity and the Elderly Trauma Patient. World J Surgery. 1996. Oct; 20(8): 1113-1119.

8. Shifflette VK, Lorenzo M, Mangram AJ, Truitt MS, Amos JD, Dunn EL: Should age be a factor to change from a Level II to a Level I trauma activation? Published in J of Trauma July 2010; Vol 69(1): p. 88-92.

9. Mangram AJ, Shifflette VK, Mitchell CD, Johnson VA, Lorenzo M, Truitt MS, Goel A, Lyons MA, Dunn EL. The creation of a geriatric trauma unit "G-60." Published in The American Surgeon. Sept 2011; Vol 77 (9): p. 1144-1146.

Manual Lorenzo MD

Professor of Surgery

Methodist Medical Center

Dallas, Texas, USA 


\section{Being elderly, a victim of trauma, is a risk factor for mortality in intensive care: Myth or Reality?}

One of the greatest challenges the trauma surgeon can encounter is the acute management of the geriatric patient. The population that is older than 60 years is exposed to specific medical-surgical situations that require a different approach in their management and treatment when compared to a younger adult population. This geriatric population is in a considerable increase worldwide. In the United States, for example, it already constitutes more than $13 \%$ of the population with a forecast of $16 \%$ in 2020 and $20 \%$ in the year 2050 or one-fifth of the population. ${ }^{1,2}$

Currently, trauma and sepsis are one of the leading causes of death within the geriatric population in the United States. In a study conducted at a level one trauma center, it was found that, within a period of twelve years, the percentage of geriatric trauma patients admitted to the hospital increased by $13 \%$ to $27 \%$ of the total Admissions of trauma patients. This was reflected in a current increase in the total number of admissions of $67 \%{ }^{3}$ The acute management of the geriatric trauma patient presents specific considerations for the health professional. The lesions in this population are of multifactorial origin and must take into consideration the physiological difficulties, environmental hazards and alterations in behavior related to the trauma of the patient. The area where these three conditions are interceded presents the group with the highest risk index for an injury. The most common cause of visits by geriatric trauma patients to the emergency room are falls. Between 65 and $70 \%$ of admissions to a trauma center by this population are due to falls. ${ }^{4}$ In one study, geriatric patients over 65 years of age had a $33 \%$ chance of falling and one $50 \%$ probability after 80 years. ${ }^{5}$ In addition, one-fifth of fatalities due to vehicle-pedestrian accidents occur in victims older than 65 years of age. ${ }^{6}$

What is the difference between the management of geriatric patients with trauma surgical urgencies and patients of younger age? Pre-existing medical conditions, compromised or diminished physiological reserve, decreased daily functional capacity, possible impairment of mental capacity, previous surgical interventions, multiple medications, domestic abuse, lack of specific needs of this population by health professionals and different patterns of injury are challenges in the management of this population. Not only do they exhibit characteristic anatomical and physiological changes, most of these patients take multiple and varied medications concomitantly. The most common medications are to control high blood pressure (55\%), antiarrhythmics (26\%), blood glucose control (22\%), antiplatelet drugs $(22 \%)$ and anticoagulants $(10 \%){ }^{7}$

The evaluation of the geriatric patient must be done in the most timely manner when arriving at the emergency room since for this medical population immediate intervention is imperative due to pre-existing medical conditions, poor physiological reserve and poor nutritional status. One of the most important factors to avoid during the evaluation of this population is the subtriage of patients with a lower or moderate injury severity index. A high degree of suspicion is necessary in the management of the geriatric patient of trauma. The medical care offered to the geriatric patient should be different from the care provided to the younger population with the same type of traumatic injuries. ${ }^{8}$ It has been found that the detailed, meticulous and early care and monitoring of these patients reduces the risk of mortality In $4.2 \% .4$

One of the most important factors in the management of the patient of geriatric trauma is to maintain a high level of medical monitoring through the hospital stay. Aggressive and constant care will increase the likelihood of these patients returning to the same physical and mental functional level prior to the traumatic event. ${ }^{9}$ This is the primary goal of the trauma team.

One of the primary considerations in the management of patients with geriatric trauma is to appreciate that these patients present an increased risk of mortality and morbidity compared to the younger adult population with similar lesions. One study found that patients with similar severity rates between the young and the geriatric population, the geriatric population with mild and moderate injury severity indices, had a fivefold increase in mortality and four times a severe index. ${ }^{8}$ In addition, The same happens with the morbidity within this population. The geriatric population showed a threefold increase between mild and moderate severity indexes and a twofold increase among severe indices as compared to the younger adult population. ${ }^{8}$ For this reason, geriatric trauma patients should be evaluated, diagnosed And handled immediately or as soon as possible upon arrival in the emergency room even if they present minor or moderate injury. Subtriaging must be avoided at all costs within this growing patient population.

We urge the authors to continue their studies and assessments in this specific population for the purpose of improving their survival rate and independent function following trauma. 


\section{REFERENCES}

1. Ortman, Jennifer M., et al. An Aging Nation: The Older Population in the United States. May 2014. p 25-1140. www.census.gov/ prod/2014pubs/p.25-1140.pdf

2. United Nations. Department of Economics an Social Affairs, populationdivision(2013).ST/ESA/SER.A/348.www.un.org/en/ development/desa/population/publications/pdf/ageing/world population ageing2013.pdf

3. Mangram AJ, Mitchell CD, Shifflette VK, Lorenzo M, Truitt MS, Goel A, Lyons MA, Nichols DJ, Dunn EL. Geriatric trauma service: a one-year experience. Published in the Journal of Trauma and Acute Care Surgery, Jan 2012; Vol 72 (1): p. 119-122.

4. Lorenzo M, Barba C, et al. Will Patient Outcomes Improve If We Admit and Manage Geriatric Patients in a Specialized Unit in the Trauma Center? Western Trauma Association Abstract Presentation. Jackson Hole, Wyoming 2005.

5. Schwab, CW, Kaunder DR, et al. Trauma in the Geriatric Patient. Arch Surg 1992;127:701.

6. Hannan EL, et al. Elderly Trauma in Patients in NY State: 1994-1998. J Trauma. 2004 Jun; 56(6):1297-1304.

7. McMahon DJ, Schawb CW, Kaunder DR. Comorbidity and the Elderly Trauma Patient. World J Surgery. 1996. Oct; 20(8): 1113-1119.

8. Shifflette VK, Lorenzo M, Mangram AJ, Truitt MS, Amos JD, Dunn EL: Should age be a factor to change from a Level II to a Level I trauma activation? Published in J of Trauma July 2010; Vol 69(1): p. 88-92.

9. Mangram AJ, Shifflette VK, Mitchell CD, Johnson VA, Lorenzo M, Truitt MS, Goel A, Lyons MA, Dunn EL. The creation of a geriatric trauma unit "G-60." Published in The American Surgeon. Sept 2011; Vol 77 (9): p. 1144-1146.

Manual Lorenzo MD

Professor of Surgery

Methodist Medical Center

Dallas, Texas, USA 